\title{
Design of single-mode waveguides for enhanced light-sound interaction in honeycomb-lattice silicon slabs
}

\author{
Jose M. Escalante, ${ }^{1, a)}$ Alejandro Martínez, ${ }^{1}$ and Vincent Laude ${ }^{2}$ \\ ${ }^{1}$ Universidad Politecnica de Valencia, Valencia, Spain \\ ${ }^{2}$ Institut FEMTO-ST, Université de Franche-Comté and CNRS, Besançon, France
}

(Received 10 December 2013; accepted 27 January 2014; published online 11 February 2014)

\begin{abstract}
We present the design of two waveguides (ladder and slot-ladder waveguides) implemented in a silicon honeycomb photonic-phononic crystal slab, which can support slow electromagnetic and elastic guided modes simultaneously. Interestingly, the photonic bandgap extends along the first Brillouin zone; so with an appropriate design, we can suppress propagation losses that arise coupling to radiative modes. From the phononic point of view, we explain the slow elastic wave effect by considering the waveguide as a chain of coupled acoustic resonators (coupled resonant acoustic waveguide), which provides the mechanism for slow elastic wave propagation. The ladder waveguide moreover supports guided phononic modes outside the phononic bandgap, similar to photonic slab modes, resulting in highly confined phononic modes propagating with low losses. Such waveguides could find important applications to the observation of optomechanical and electrostriction effects, as well as to enhanced stimulated Brillouin scattering and other opto-acoustical effects in nanoscale silicon structures. We also suggest that they can be the basis for a "perfect" photonic-phononic cavity in which damping by coupling to the surroundings is completely forbidden. (C) 2014 AIP Publishing LLC. [http://dx.doi.org/10.1063/1.4864661]
\end{abstract}

\section{INTRODUCTION}

The possibility to open simultaneous photonic and phononic bandgaps in certain periodic structures has been recently suggested. ${ }^{1-3}$ These structures, called phoxonic or optomechanical crystals, display forbidden bands for both photons and phonons simultaneously, referred to as phoxonic bandgaps. $^{2-4}$ The realization of periodic structures, where light and sound can be controlled simultaneously, would allow the observation of interesting phenomena such as the strong confinement and light-sound interaction in very small volumes, ${ }^{5}$ the reduction of the group velocity for both types of waves simultaneously, ${ }^{6}$ or the strong enhancement of stimulated Brillouin scattering. ${ }^{7}$

In order to have a full control over light and sound in all directions, phoxonic crystals should be ideally threedimensional (3D). ${ }^{8,9}$ However, the physical implementation of $3 \mathrm{D}$ photonic, phononic, or phoxonic structures is quite complicated, and even more is the introduction of defects, such as cavities or guides. A more practical alternative to $3 \mathrm{D}$ structures is the use of two-dimensional (2D) periodic structures in a suspended slab. ${ }^{4}$ It has been shown recently that a thin silicon slab perforated by an array of cylindrical holes (arranged in square or honeycomb lattices) can support phoxonic bandgaps for modes confined in the slab. ${ }^{4}$ Point and linear defects in such 2D phoxonic crystal slabs would allow the simultaneous confinement of light and sound in nanoscale volumes. ${ }^{5,6,10}$ Interestingly, silicon phoxonic crystal structures can be fabricated using mainstream CMOS technology, which opens the door towards large-scale production of nanoscale devices supporting light and sound interactions.

\footnotetext{
a)jmescalantefernadez@gmail.com
}

An interesting property of photonic crystal waveguides is the possibility to significantly decrease the speed of light. Indeed, slow-light propagation in photonic crystal waveguides has a great potential to enhance enormously nonlinear processes owing to both slow propagation and highly confined fields inside the structure. ${ }^{11}$ Development of phoxonic waveguides supporting the propagation of slow photons and phonons at the same time would allow for observing interesting effects resulting from enhanced photon-phonon interaction, confirming the importance of the development of this kind of waveguides.

A key point for future applications and devices based on the interaction between light and sound waves is to reduce (and ultimately avoid) propagation losses. From the photonic point of view, and for wavelengths in the telecom window, where silicon is nearly transparent, the main source of optical losses in waveguides arises from the coupling of scattered light by fabrication defects to radiative modes placed above the light line. From the phononic point of view, the main source of losses comes from coupling with thermal phonons because of anharmonicity, which is an intrinsic source of losses of the material. A second source of losses, in addition, arises from fabrication defects. The existence of a unique phononic mode inside the bandgap would reduce losses since coupling to other modes by scattering in fabrication defects would be completely neglectable, resulting in a waveguide, whose losses are only due to intrinsic material losses. Furthermore, designed waveguides in which electromagnetic and elastic waves are tightly confined at the same time should allow observing an enhancement of their interaction.

In this work, we present two phoxonic crystal waveguides, which support simultaneously photonic and phononic slow modes. The waveguides are created by inserting line 
defects in a 2D silicon slab perforated by a honeycomblattice of circular holes. Interestingly, the photonic bandgap for guided modes in the honeycomb lattice extends over the whole Brillouin zone and beyond the light line. In this sense, we show that some linear defects support guided modes in a frequency region, where no radiative modes are present, which would significantly reduce propagation losses. For the case of phonons, we obtain phononic guided modes inside the phononic bandgap, which can be explained in the context of coupled resonators acoustic waveguide (CRAW), ${ }^{12,13}$ but also phononic modes below the first phononic band of the perfect crystal (Lamb or plate modes), which are similar to photonic slab modes. ${ }^{14,15}$

\section{WAVEGUIDES IN THE HONEYCOMB-LATTICE SLAB}

A honeycomb lattice of circular holes on a silicon slab provides a photonic and phononic (i.e., phoxonic) bandgaps for guided modes by properly choosing the parameters. ${ }^{8}$ In this work, we have chosen the following parameters: $h=0.5 a$ (slab thickness) and $r=0.25 a$ (hole radius), where $a=690 \mathrm{~nm}$ is the lattice constant. To obtain the dispersion relation for the photonic and phononic crystals, we have used two different numerical methods: plane wave expansion method (PWE) for photonic bands and finite element method (FEM) for phononic bands. The PWE method has not been used in the phononic case due to the convergence problem for structures with large elastic mismatch. The dimensions of the super-cell, when defects are added, have been properly chosen to avoid interaction between neighboring waveguides. In the photonic case, vertical coupling between super-cells was avoided by checking that the photonic bandgap width remains unchanged when the air thickness between super-cells is increased.

From the photonic point of view, the honeycomb lattice has a great interest since the photonic band gap extends inside the light cone, whereas typical band gaps in 2D photonic crystal slabs only extend outside the light cone (i.e., they apply only to truly guided modes). ${ }^{6,10}$ It is expected that the absence of radiative modes in the gap region will inhibit the strong loss mechanism resulting from the scattering of light propagating through waveguides by defects (arising from fabrication imperfections) and its subsequent coupling to radiative modes. From the phononic point of view, the wide and complete phononic band gap exists for a significant range of thickness and filling factors. It thus provides us with a robust way to create phononic waveguides in which acoustic structural attenuation is strongly suppressed, since there are no other modes to which scattered phonons can couple. ${ }^{6,10}$

We consider two types of linear defects that are designed to support slow photonic (only for odd-parity) and phononic modes within their respective band gaps. Fig. 1 displays the two waveguides. The width of the core is $\Delta=\alpha d$, with $d=a / \sqrt{3}$, and $\alpha$ is a design parameter. $H$ is the width of the additional slot considered for the second waveguide. The radius of the two holes adjacent to the defect, $r^{\prime}=\beta r(\beta<1)$, is also adjusted.
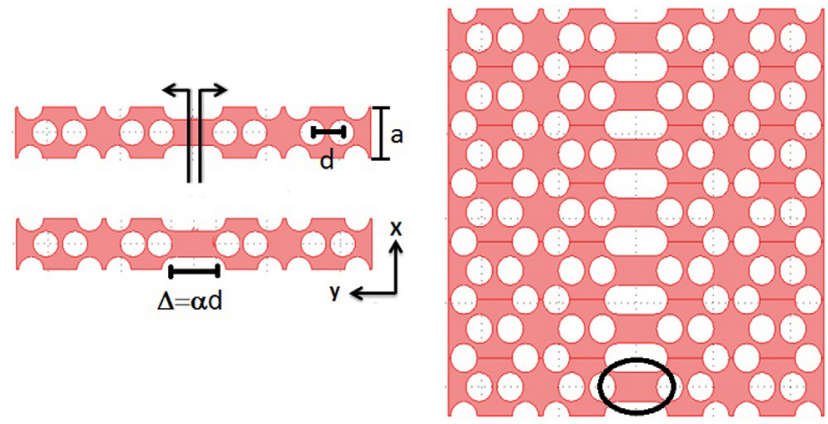

(a)

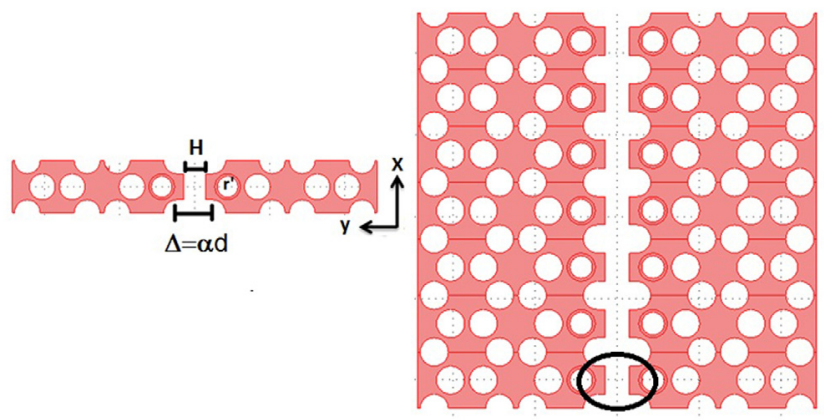

(b)

FIG. 1. Proposed linear defects created on a honeycomb lattice silicon phoxonic crystal slab: (a) ladder waveguide and (b) slot-ladder waveguide. The black ellipses indicate the micromechanical resonator in each structure.

\section{A. Ladder waveguide}

With the ladder waveguide shown in Fig. 1(a), we pursue two goals. First, from a photonic point of view, we wish to form a single-mode waveguide by decreasing the effective index in the defect region so that the photonic guided modes will come from the dielectric band (i.e., bands below the photonic bandgap). Second, from a phononic point of view, the steps of the ladder waveguide are not connected to one another but are instead left free to vibrate, working as micromechanical resonators. As mechanical vibrations can only propagate via the surrounding crystal, a CRAW is formed, ${ }^{12,13}$ allowing slow phononic slow modes to propagate at frequencies inside the bandgap.

Figures 2(a) and 2(b) display the photonic and the phononic dispersion relations of the ladder waveguide, for $\alpha=1.2$. A single guided band extends inside both bandgaps. Interestingly, both guided bands are very flat, which is a signature of slow-wave propagation (slow guided modes). The group velocity of the guided phononic and photonic modes is plotted in Figs. 3(a) and 3(b). Notice that the group velocity for phononic waves is normalized with respect to $v=8433 \mathrm{~m} / \mathrm{s}$, which is the sound velocity at $20^{\circ} \mathrm{C}$ in thin silicon rods, i.e., rods with sub-wavelength diameter. ${ }^{16,17}$

There is a region of $k$-space, where the phononic group velocity is close to zero (for $k a / 2 \pi \geq 0.35$ ). Concurrently, the photonic group velocity of the guided mode is reduced by at least two orders of magnitude. Although the photonic bandgap is empty of extended photonic modes above the light cone, however, it can be seen from Figs. 2(a) and 3(b) that the guided mode appearing inside the bandgap also 


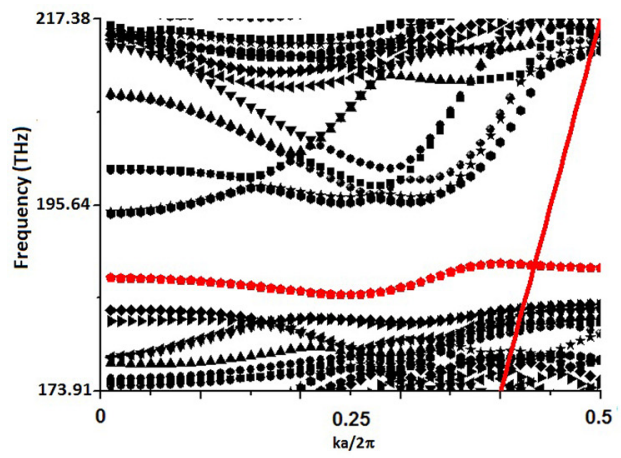

(a)

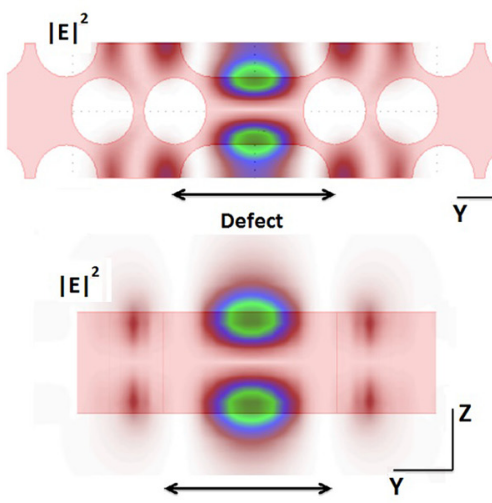

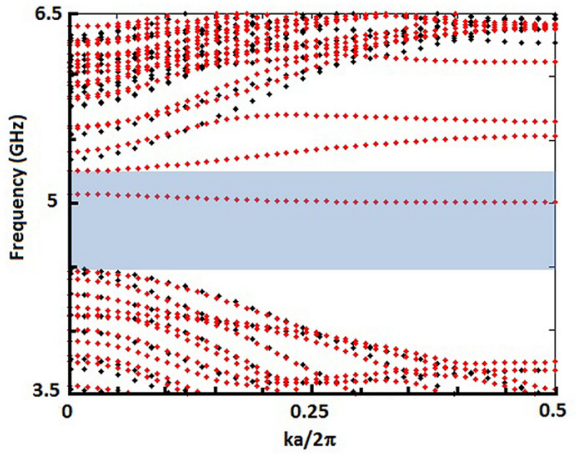

(b)

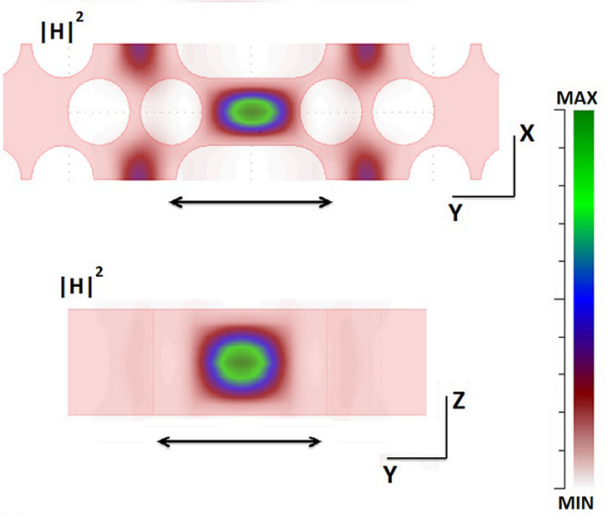

(c)

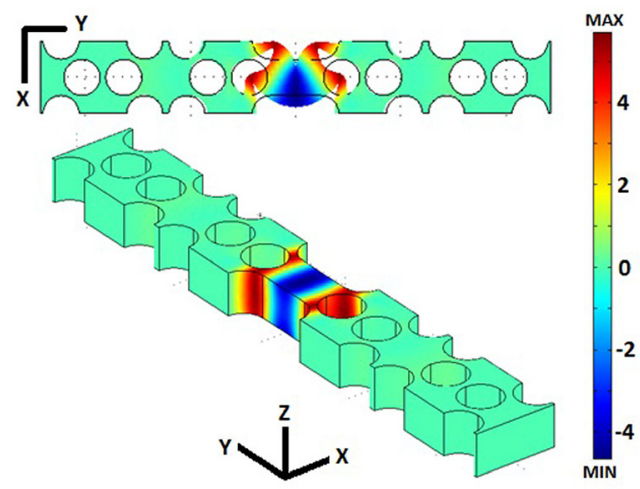

(d)

extends into the radiative zone in the same frequency range. This means that the goal of completely eliminating radiative modes in the region of interest is not achieved. Actually, fabrication defects could easily scatter light from the lossless guided mode to the leaky guided mode in the radiative region, owing to their similar wave vectors and to the high confinement in the defective region. ${ }^{18}$ The slot-ladder waveguide described in Sec. II B is free of this shortcoming.

From the phononic point of view, the ladder waveguide shows a very interesting behavior. It supports two different kinds of guided modes: bandgap modes (modes inside the phononic bandgap) and slab modes (see Fig. 4). The phononic slow guided modes that appear inside the phononic bandgap (see Fig. 2(b), blue region) can be explained, if we consider the waveguide as a linear chain of coupled micromechanical resonators. $^{12,13}$ The micromechanical resonators are here the steps of the ladder. Considering the theoretical expression for the dispersion relation of guided modes of a CRAW, ${ }^{13}$ we get an adequate convergence with only a few harmonic coefficients (see Table I). In contrast, the phononic modes appearing below the first extended phononic band (slab modes, see Fig. 4(a)) are similar to photonic slab modes. ${ }^{14,15}$ In this case, the guiding mechanism is not the existence of a phononic bandgap. Instead, guided modes are similar to Lamb waves of the suspended silicon step of the ladder. We find two different phononic slab modes, with regards to how the field is confined within the defect: they can either be confined around the linear defect (Fig. 4(b)), which look like a symmetric Lamb mode trapped between two semi-infinite phononic crystal mirrors (two surface modes coupled through the silicon bridge), or confined inside the defect (Fig. 4(c)), which is similar to photonic slab modes in terms of energy confinement.

The phononic mode inside the bandgap displays a very slow group velocity, as discussed earlier. It induces more 


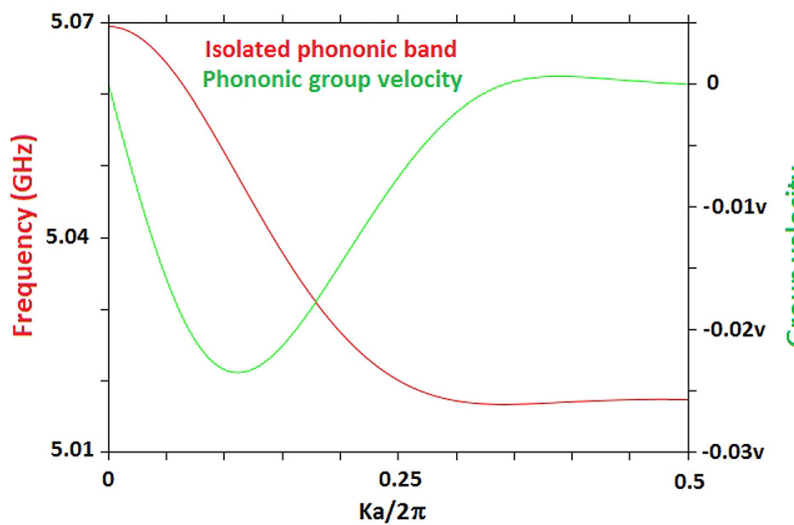

(a)

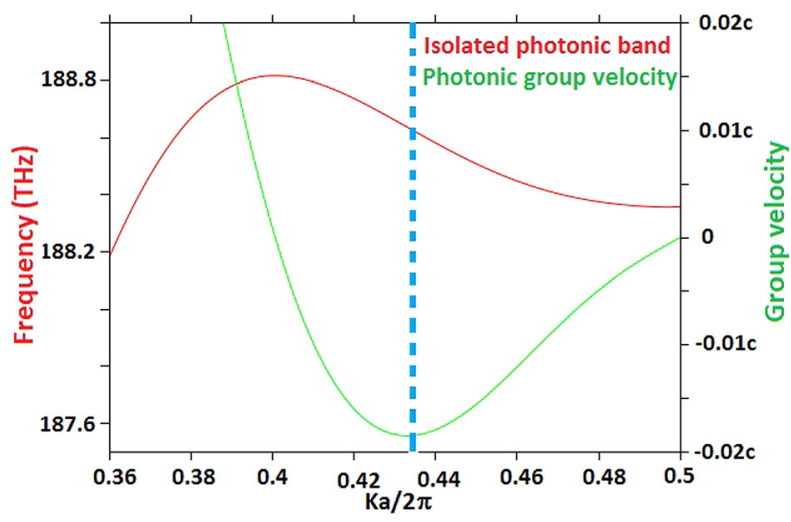

(b)

FIG. 3. Ladder waveguide: (a) Phononic dispersion relation and group velocity and (b) photonic dispersion relation and group velocity for $\alpha=1.2 \mathrm{c}$ is the celerity of light in a vacuum and $v=8433 \mathrm{~m} / \mathrm{s}$ is the sound velocity at $20^{\circ} \mathrm{C}$ in thin silicon rods. The blue dashed line in (b) is the air light line.

strain in the core of the photonic waveguide than slab modes do, and therefore, would be, in principle, more adequate for enhanced photoelastic coupling. Slab modes, in turn, induce a larger displacement of the interfaces of the silicon bridges, and would, in principle, be more adequate for optomechanical coupling based on moving interface coupling.

\section{B. Slot-ladder waveguide}

Upon introducing the slot-ladder waveguide of Fig. 1(b), we are trying to remove the photonic radiative modes that appear inside the photonic bandgap, whilst retaining the other properties of the ladder waveguide.

Figures 5(a) and 5(b) display the photonic and phononic band structures of the slot-ladder waveguide for $\alpha=1.2$, $H=300 \mathrm{~nm}$, and $\beta=0.8$. It can be seen that there are still a single photonic and a single phononic slow guided modes appearing inside the bandgaps. As apparent in Fig. 6(b), the $k$-range, where the phononic group velocity is close to zero, is reduced due to the presence of the slot $(k a / 2 \pi \geq 0.45)$. Furthermore, in comparison to the ladder waveguide, the photonic group velocity of the guided mode, shown in Fig. $6(\mathrm{a})$, is only reduced by one order of magnitude. The most important property of this waveguide, however, is that the radiative modes above the light cone have been removed,

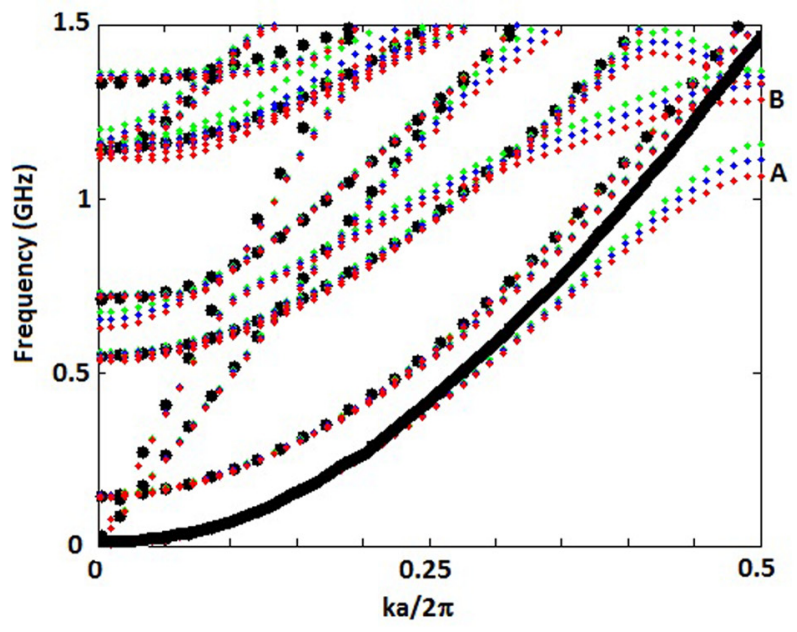

(a)

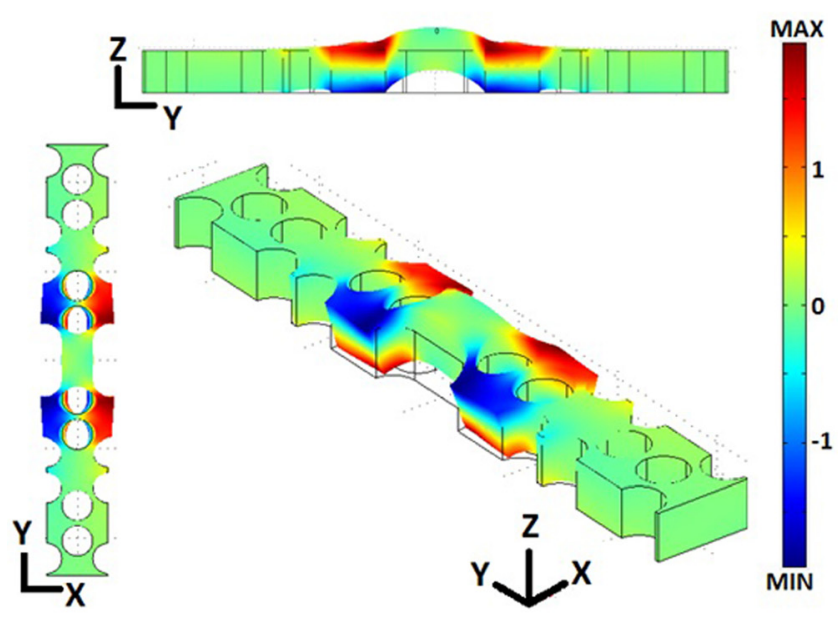

(b)

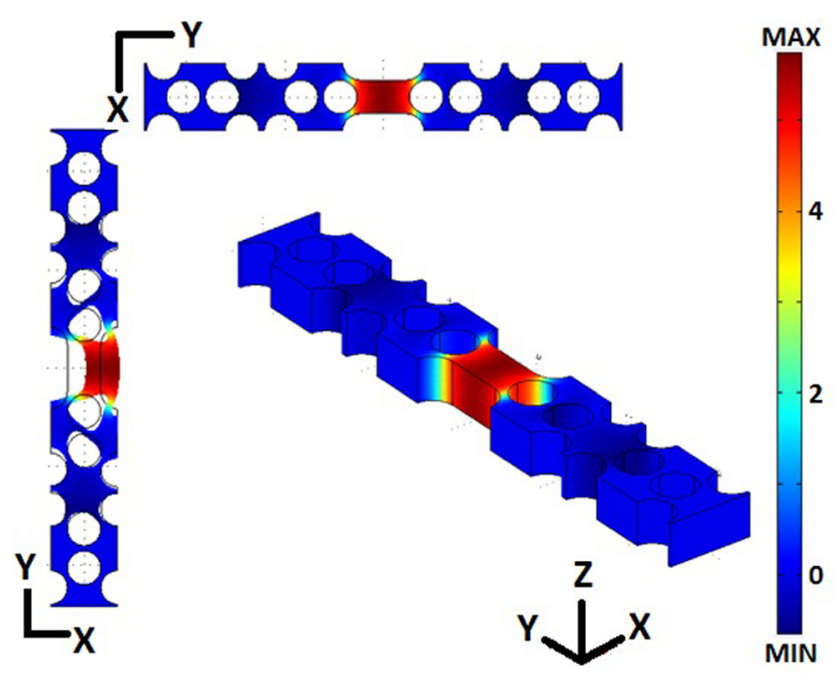

(c)

FIG. 4. Phononic slab modes of the ladder waveguide. (a) Guided modes appear below the first band of defect-less phononic crystal. Phononic band structures are plotted without defect (big black dots), and with defect for $\alpha=1$ (small green dots), $\alpha=1.1$ (small blue dots), and $\alpha=1.2$ (small red dots). (b) Displacement field pattern at point A and (c) displacement field pattern at point $\mathrm{B}$ of the band structure for $\alpha=1.2$. 
TABLE I. Fourier coefficients of the CRAW phononic dispersion relation for the ladder and the slot-ladder waveguides. ${ }^{\text {a }}$

\begin{tabular}{lcrrrr}
\hline \hline Waveguide & $\Gamma_{0}(\mathrm{GHz})$ & $\Gamma_{1}(\mathrm{GHz})$ & $\Gamma_{2}(\mathrm{GHz})$ & $\Gamma_{3}(\mathrm{GHz})$ & $\Gamma_{4}(\mathrm{GHz})$ \\
\hline Ladder & 5.03125 & 0.02292 & 0.01169 & 0.00299 & 0.00048 \\
Slot-ladder & 4.91223 & -0.06004 & -0.00774 & 0.00542 & 0.00079 \\
\hline \hline
\end{tabular}

Theoretical expression of dispersion relation for a CRAW: ${ }^{13}$ $\omega=\Gamma_{0}+\sum_{m=1}^{\infty} \Gamma_{m} \cos (m k a)$.

eliminating the source of losses due to the coupling of scattered light to leaky guided modes.

As before, the existence of a slow phononic slow guided mode for frequencies inside the phononic bandgap can be explained by considering the waveguide as a linear chain of coupled micromechanical resonators. The coefficients of the
CRAW dispersion relation of the phononic guided mode are given in Table I. The displacement field pattern in Fig. 5(d) is similar to that of a Lamb wave propagating with one side clamped (on the phononic crystal side) and one side free (on the slot side).

Interestingly, the slot-ladder waveguide could be used to form an optomechanical cavity using the heterostructure approach. ${ }^{19}$ Photons can be well confined inside the defect region and a very high $\mathrm{Q}$ factor can be reached due to the fact that cavity photons cannot couple to radiative modes over the light line. ${ }^{20}$ In the case of phonons, and for measurements performed in a vacuum environment, the situation is similar: there are no radiative paths for phonons to leave the cavity. In this sense, such a phoxonic crystal cavity could improve over optomechanical cavities having a phononic bandgap only for some symmetry and that require an

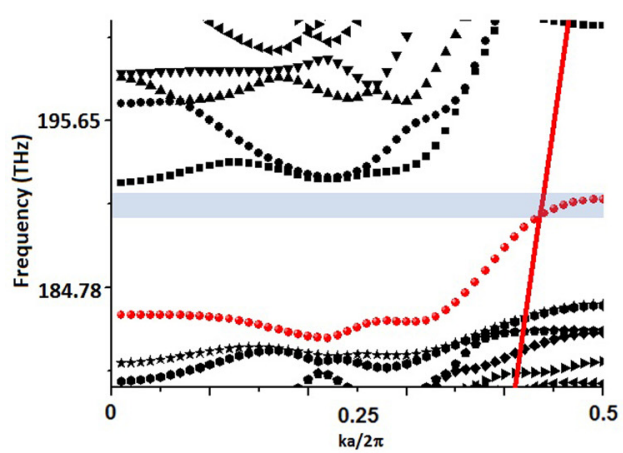

(a)

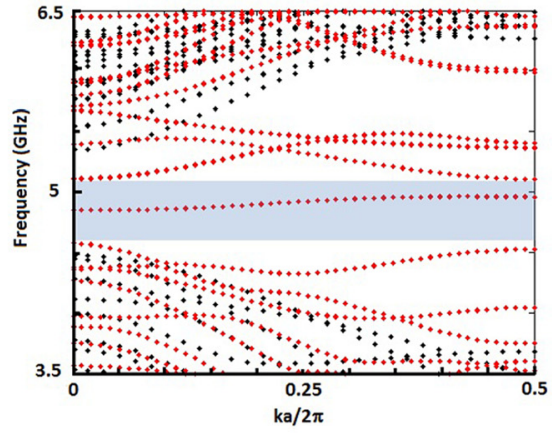

(b)
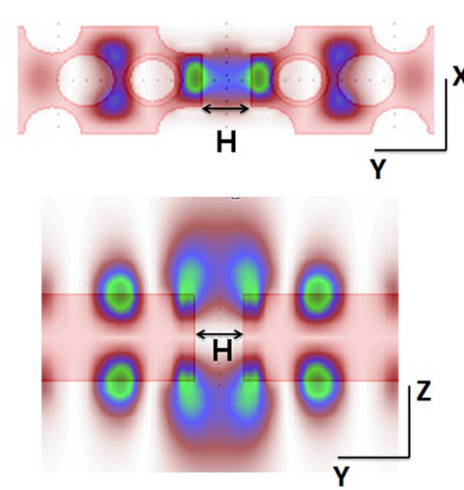

$\mathbf{x}$
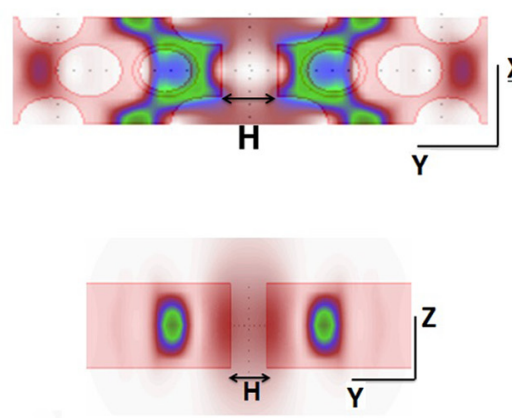

(c)

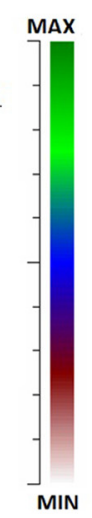

FIG. 5. Slot-ladder waveguide: (a) Photonic band structure. The blue region indicates the range of frequencies free of radiative losses for guided modes, (b) phononic band structure, (c) electric and magnetic field patterns of the photonic guided mode $(k a / 2 \pi=0.5)$, and (d) displacement field pattern of the phononic guided mode $(k a / 2 \pi=0.5)$. All plots are for $\alpha=1.2, H=300 \mathrm{~nm}$, and $\beta=0.8$.

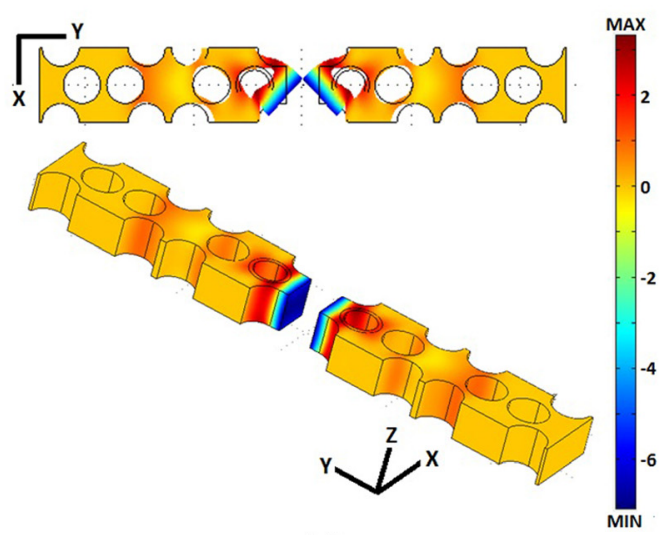

(d) 


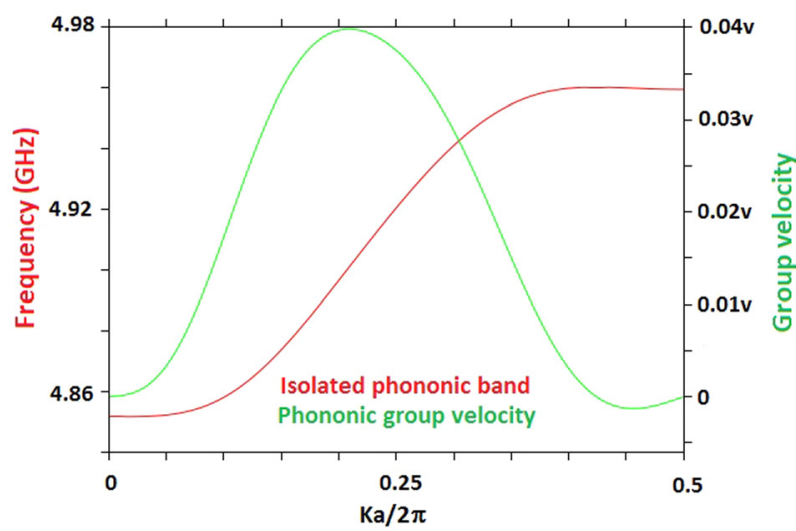

(a)

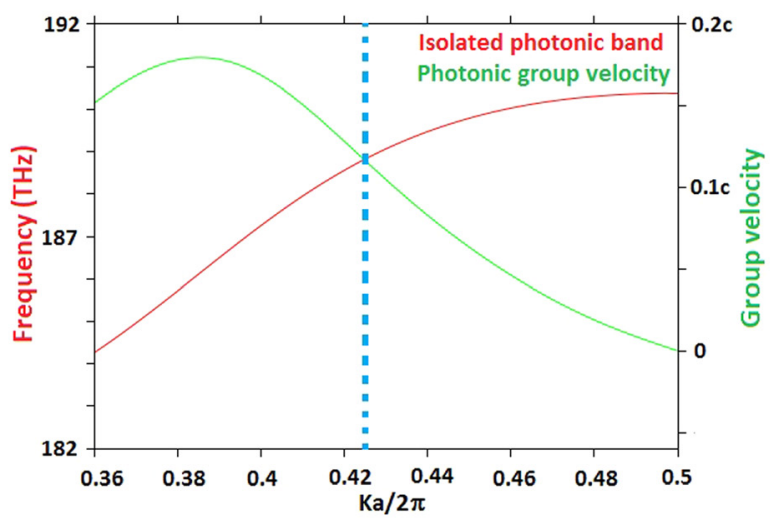

(b)

FIG. 6. Slot-ladder waveguide: (a) Phononic dispersion and group velocity and (b) photonic dispersion relation and group velocity for $\alpha=1.2$, $H=300 \mathrm{~nm}$, and $\beta=0.8 . c$ is the celerity of light in a vacuum and $v=8433 \mathrm{~m} / \mathrm{s}$ is the sound velocity at $20^{\circ} \mathrm{C}$ in thin silicon rods.

external phononic shield to avoid phonon damping. ${ }^{21}$ Therefore, such a 2D cavity could be extremely interesting to observe strong light-sound coupling at the nanoscale.

In comparison to the previous phoxonic crystal waveguide design reported in Ref. 6, our designs here display some advantages, namely, the absence of photonic radiative modes over the light line, which should reduce photonic propagation losses, and the existence of a single slow phononic mode inside the phononic bandgap, so that intermodal coupling can be neglected. From the point of view of fabrication, both square- and honey-comb lattice silicon phoxonic crystals make use of non-standard silicon slabs with a $400 \mathrm{~nm}$ thickness (the standard silicon thicknesses in siliconon-insulator wafers are 220 and $250 \mathrm{~nm}$ ). However, light guidance in honeycomb-lattice photonic crystal waveguides with dimensions similar to those we have employed in our design has been recently demonstrated experimentally. ${ }^{18}$

\section{CONCLUSION}

In this work, we have presented two novel photonicphononic slow waveguides defined in a silicon honeycomb phoxonic crystal slab. We have demonstrated the existence of single-mode low-group velocity acoustic and optical guidance for frequencies within phononic and photonic bandgaps.
The strong confinement of both waves in the waveguide, together with their low group velocities, should result in the reduction of the propagation distances and threshold powers to observe Brillouin-like effects for on-chip guided waves. ${ }^{22}$ These results are scalable to a desired frequency range by adjusting the lattice constant of the phoxonic crystal.

So far, most results regarding optomechanical interactions have been obtained in cavities (optomechanical cavities). We point at the interest of considering a new kind of optomechanical structure, the optomechanical waveguide, such as the one recently demonstrated, ${ }^{22}$ but with the added benefit of supporting slow-wave propagation for both light and sound. ${ }^{6}$ We also suggest that the slot-ladder waveguide could be used to build a heterostructure optomechanical cavity with reduced optical and acoustic losses, which could be used for sideband cooling of the cavity down to its quantum mechanical ground state. ${ }^{23}$

\section{ACKNOWLEDGMENTS}

Financial support from the multidisciplinary project of UPV, PAID-05-12 (CE 20130141).

${ }^{1}$ M. Maldovan and E. L. Thomas, Appl. Phys. Lett. 88(25), 251907 (2006).

${ }^{2}$ M. Maldovan and E. L. Thomas, Appl. Phys. B 83(4), 595-600 (2006).

${ }^{3}$ S. Sadat-Saleh, S. Benchabane, F. I. Baida, M. P. Bernal, and V. Laude, J. Appl. Phys. 106, 074912 (2009).

${ }^{4}$ S. Mohammadi, A. A. Eftekhar, A. Khelif, and A. Adibi, Opt. Express 18(9), 9164-9172 (2010).

${ }^{5}$ M. Eichenfield, J. Chan, R. M. Camacho, K. J. Vahala, and O. Painter, Nature 462, 78-82 (2009).

${ }^{6}$ V. Laude, J. C. Beugnot, S. Benchabane, Y. Pennec, B. Djafari-Rouhani, N. Papanikolaou, J. M. Escalante, and A. Martinez, Opt. Express 19, 9690 (2011).

${ }^{7}$ W. Qiu, P. T. Rakich, M. Soljacic, and Z. Wang, e-print arXiv:1210.0738. ${ }^{8}$ Y. Pennec, B. D. Rouhani, E. H. E. Boudouti, C. Li, Y. E. Hassouani, J. O. Vasseur, N. Papanikolaou, S. Benchabane, V. Laude, and A. Martinez, Opt. Express 18, 14301 (2010).

${ }^{9}$ N. Papanikolaou, I. E. Psarobas, and N. Stefanou, Appl. Phys. Lett. 96, 231917 (2010).

${ }^{10}$ Y. Pennec, B. D. Rouhani, E. H. E. Boudouti, C. Li, Y. E. Hassouani, J. O. Vasseur, N. Papanikolaou, S. Benchabane, V. Laude, and A. Martinez, Chin. J. Phys. 49(1), 100 (2011).

${ }^{11}$ M. Soljacic and J. D. Joannopoulos, Nature Mater. 3, 211 (2004).

${ }^{12}$ A. Khelif, S. Mohammadi, A. A. Eftekhar, A. Adibi, and B. Aoubiza, J. Appl. Phys. 108, 084515 (2010)

${ }^{13}$ J. M. Escalante, A. Martínez, and V. Laude, J. Phys. D: Appl. Phys. 46, 475301 (2013).

${ }^{14}$ A. Adibi, Y. Xu, R. K. Lee, A. Yariv, and A. Scherer, J. Lightwave Technol. 18(11), 1554 (2000)

${ }^{15}$ A. Adibi, Y. Xu, R. K. Lee, A. Yariv, and A. Scherer, Phys. Rev. B 64(3), 033308 (2001).

${ }^{16}$ L. E. Kinsler et al., Fundamentals of Acoustics, 4th ed. (John Wiley and sons Inc., New York, USA, 2000).

${ }^{17}$ J. Krautkramer and H. Krautkrämer, Ultrasonic Testing of Materials, 4th ed. (Springer-Verlag, Berlin, Germany, 1990), p. 497.

${ }^{18}$ D. Puerto, A. Griol, J. M. Escalante, Y. Pennec, B. D. Rouhani, J.-C. Beugnot, V. Laude, and A. Martinez, IEEE Photon. Technol. Lett. 24(22), 2056 (2012).

${ }^{19}$ B. S. Song, S. Noda, T. Asano, and Y. Akahane, Nature Mater. 4, 207 (2005).

${ }^{20}$ A. H. Safavi-Naeini, T. P. M. Alegre, M. Winger, and O. Painter, Appl. Phys. Lett. 97, 181106 (2010).

${ }^{21}$ J. Chan, A. H. Safavi-Naeini, J. T. Hill, S. Meenehan, and O. Painter, Appl. Phys. Lett. 101, 081115 (2012).

${ }^{22}$ H. Shin, W. Qiu, R. Jarecki, J. A. Cox, R. H. Olsson III, A. Starbuck, Z. Wang, and P. T. Rakich, Nature Commun. 4, 1944 (2013).

${ }^{23}$ J. Chan, T. P. M. Alegre, A. H. Safavi-Naeini, J. T. Hill, A. Krause, S. Gröblacher, M. Aspelmeyer, and O. Painter, Nature 478, 89 (2011). 